\title{
Diagnosis of Pneumocystis carinii pneumonia from non-invasive sampling of respiratory secretions
}

\author{
R A Hague, S E Burns, J Y Q Mok, P L Yap
}

\begin{abstract}
An infant infected with HIV presented with fever, tachypnoea, hypoxia, and radiological evidence of bilateral pneumonitis. Fluorescent antibody technique identified Pneumocystis carinii within 24 hours from secretions obtained by nasopharyngeal aspiration. This rapid, non-invasive method should be the first line investigation of suspected $P$ carinii pneumonia in immunocompromised patients.
\end{abstract}

Pneumocystis carinii is an important pathogen in the immunocompromised child, and must be considered in the differential diagnosis of any such child presenting with respiratory symptoms. Characteristic radiological appearances are of diffuse reticulogranular infiltrates, particularly in the perihilar region, progressing to consolidation, but a normal chest radiograph is compatible with the diagnosis. ${ }^{1}$ Cytomegalovirus or mycobacteria infection may produce similar findings.

Definitive diagnosis has previously relied on the demonstration of silver staining cysts in material obtained from open lung biopsy or bronchioalveolar lavage, requiring an invasive procedure in an acutely ill child. ${ }^{23}$ Sputum induction in infants and small children is not a practicable technique, ${ }^{4}$ and serology is unhelpful. ${ }^{5}$ We report a case in whom the

diagnosis was made from nasopharyngeal secretions using a fluorescent antibody technique.

\section{Case report}

A white infant girl whose HIV seropositive mother developed $P$ carinii pneumonia at 35 weeks' gestation was delivered at term weighing $3050 \mathrm{~g}$. There were no neonatal problems, and her initial progress was complicated only by persistent candidal infection of the oral cavity and napkin area.

At 4.5 months she presented with a two week history of mild upper respiratory symptoms, two days of fever, an unproductive cough, and increasing tachypnoea, sufficiently severe to impair feeding. Examination showed a pale, mildly cyanosed and lethargic, but responsive infant. She had a tachycardia of 160 /minute and respiratory rate of $80 /$ minute, with bilateral intercostal and subcostal indrawing and scattered crepitations on auscultation. Lymphadenopathy and hepatosplenomegaly were noted and there was candidal dermatitis of the napkin area.

A chest radiograph showed extensive bilateral pneumonitis (figure). She was hypoxic (oxygen saturation $60 \%$ ), with a carbon dioxide tension $\left(\mathrm{PCO}_{2}\right)$ of $5.37 \mathrm{kPa}$ and hydrogen ion concentration of $39.7 \mathrm{mmol} / \mathrm{l}$. On admission the neutrophil count was $6200 \times 10^{6} / 1$, T4 lymphocyte count $1079 \times 10^{6} / 1$, and T4/T8 ratio $1 \cdot 4$. Serum IgG concentration was normal $(4 \mathrm{~g} / \mathrm{l})$, and $\operatorname{IgA}$ and $\operatorname{IgM}$ raised $(0.9 \mathrm{~g} / \mathrm{l}$ and $2 \cdot 1 \mathrm{~g} / \mathrm{l})$. HIV p24 antigen was positive $(140 \mathrm{pg} / \mathrm{ml})$. Secretions obtained by nasopharyngeal aspiration were sent for microbiological investigation.

Pending results the infant received oxygen supplementation and high dose cotrimoxazole and acyclovir intravenously. The identification of $P$ carinii was confirmed the next day (see below). The infant's condition initially stabilised, but deteriorated 24 hours later. Despite giving hydrocortisone and intravenous immunoglobulin, she failed to maintain oxygen saturation of $>80 \%$ even with fractional inspiratory oxygen of $100 \%$, and developed carbon dioxide retention $\left(\mathrm{PCO}_{2} 9 \cdot 75 \mathrm{kPa}\right)$ and respiratory acidosis (hydrogen ion concentration 73.7 $\mathrm{mmol} / \mathrm{l})$. Definite knowledge of the diagnosis, and its implications for the child's long term prognosis meant that the parents could be counselled appropriately. Their wish for her not to receive mechanical ventilation was respected, and she died on the fourth day of her admission. Postmortem examination confirmed the widespread alveolar exudate within the 
by silver staining. No other organisms were detected.

\section{Method}

After addition of an equal volume of sputolysin (Behring Diagnositics) and glass beads the sample was vortexed for one minute and the incubated at $37^{\circ} \mathrm{C}$ for three minutes. A total of $10 \mathrm{ml}$ of phosphate buffered saline (PBS) $\mathrm{pH}$ $7 \cdot 2$ was added and the mixture centrifuged at $2500 \mathrm{~g}$ for 20 minutes. The pellet was resuspended in $1 \mathrm{ml}$ PBS and cytospin preparations made on two polyl-lysine coated slides. A cytospin preparation of positive rat lavage material was used as a positive control. After fixation for 30 minutes in equal volumes $(50 \%)$ methanol and acetone, trypsin $(0 \cdot 25 \%)$ digestion for 10 minutes at $37^{\circ} \mathrm{C}$ followed. A standard wash procedure of three five minutes with PBS was carried out.

Mouse monoclonal antibody to $P$ carinii (Northumbria Biologicals Limited) was added to the test and positive control slides and PBS only, and incubated for $\mathbf{4 0}$ minutes. After repeat washing antimouse IgG (F'ab) conjugated to fluorescein isothiocyanate was added and a further incubation of 40 minutes followed. After a last wash procedure substituting PBS pH 8.4 for the final change, the slides were mounted and viewed under ultraviolet light.

The sample was considered positive when three typical cysts showing all over fluorescence were seen, further confirmed by methenane silver treatment of the control slide.

\section{Discussion}

In HIV infection, the diagnosis of $P$ carinii pneumonia has important implications, not only for the treatment of the acute illness, but also for longer term prognosis. In New York 13 out of 15 children less than 6 months old with $P$ carinii pneumonia died during the acute episode, and all were dead by one year ( $J$ Chow, $K$
Shah, $\mathrm{K} \mathrm{Li}$, et al; abstract presented at $\mathrm{V}$ International Conference on AIDS, Montreal, June 1989. Because of the risks involved in, and the technical expertise required for, paediatric bronchoscopy treatment is usually empirical. If the child dies, postmortem examination may give the answers, but if the child responds to high dose cotrimoxazole, the differential diagnosis between a bacterial pneumonia and $P$ carinii pneumonia remains unresolved. This then creates difficulties in classification and staging of the child's disease, and in making therapeutic decisions, such as whether to commence zidovudine or prophylaxis for $P$ carinii pneumonia.

Identification of $P$ carinii pneumonia from upper respiratory tract secretions may only be possible in cases of overwhelming infection, and failure to detect it by this method does not exclude the diagnosis. It is in the most severe cases, however, that the patient is least able to tolerate more invasive procedures. Because our technique is rapid, other methods can still be used thereafter without undue delay. We believe therefore that it should be used as the first line investigation of suspected $P$ carinii pneumonia.

RAH is supported by the Medical Research Council. We thank the staff in wards 7A and 15 at the City Hospital for their help and support.

1 Barter S. The radiology of AIDS. Br $\mathcal{F}$ Hosp Med 1987;38: $214-8$.

2 Wolff LJ, Bartlett MS, Baehner RL, et al. The causes of interstitial pneumonia in immunocompromised children: an aggressive systematic approach to diagnosis. Pediatrics an aggressive

3 De Blic J, McKelvie P, Le Bourgeois $M$, et al. Value of bronchoalveolar lavage in the management of severe acute bronchoalveolar lavage in the management of severe acute pneumonia and interstitial pneumonitis in

4 Zaman MK, Wooten OJ, Suprahmanya B, et al. Rapid noninvasive diagnosis of Pneumocystis carinii from induced liquefied sputum. Ann Intern Med 1988;109:7-10

5 Williford Pifer LL, Woods DR, Edwards CC, et al. Pneumocystis carinii serologic studies in pediatric acquired immunodefiency syndomre. Am $\mathcal{F}$ Dis Child 1988;142:36-
The Royal Liverpool Children's Hospital Alder Hey

A Mark Dalzell Hendrik $K$ F van Saene David P Heaf

Correspondence to: Dr A Mark Dalzell, Hospital Herston Road,

Brisbane,

Queensland, 4029, Australia.

Accepted 5 July 1990

(Anch Dis Child 1990;65:1365-7).

\title{
Cystic fibrosis, Pseudomonas aeruginosa, and selective decontamination
}

\author{
A Mark Dalzell, Hendrik K F van Saene, David P Heaf
}

\author{
Abstract \\ We used an oral topical antibiotic preparation \\ to try and prevent oropharyngeal carriage of \\ Pseudomonas aeruginosa in patients with \\ cystic fibrosis. Ten of 15 patients treated with \\ a two week course of intravenous ceftazidime \\ together with a 90 day course of an antibiotic \\ containing gel continued to carry $P$ aerugi- \\ nosa in the oropharynx.
}

The technique of selective decontamination of the digestive system uses oral antibiotic combinations to prevent the overgrowth of certain groups of organism (typically Gram negative aerobes), and allows the more usual flora to maintain colonisation resistance. ${ }^{1}$ It has application particularly in the immunocompromised patient undergoing cytotoxic chemotherapy and in long term ventilated patients, ${ }^{2}$ where Gram 